\title{
Azathioprine dosing and metabolite measurement in pediatric inflammatory bowel disease: does one size fit all?
}

\author{
Rebecca Walker, Jochen Kammermeier, Rakesh Vora, Mohamed Mutalib
}

Evelina London Children's Hospital, London, UK

\section{Abstract}

\section{Introduction}

Inflammatory bowel disease (IBD) is a chronic, immuneregulated inflammatory condition of the digestive system. It is thought to have a complex etiology involving genetic, immunological and environmental factors [1]. The worldwide incidence and prevalence of IBD in children is increasing: the current incidence of Crohn's disease (CD) in children ranges between 2.5 and 11.4/100,000 [2]. Pediatric IBD is associated

Department of Paediatric Gastroenterology, Evelina London Children's Hospital, London, UK

Conflict of Interest: None

Correspondence to: Dr Rebecca Walker, Department of Paediatric Gastroenterology, Evelina London Children' Hospital, St Thomas' Hospital, Westminster Bridge Road, London SE1 7EH, UK, e-mail: r.walker7@nhs.net

Received 25 January 2019; accepted 9 April 2019; published online 10 May 2019

DOI: https://doi.org/10.20524/aog.2019.0381 with more extensive and aggressive disease that has higher rates of surgical management $[3,4]$. The main treatment goals in IBD management include timely and effective induction of remission followed by maintenance, with crucial focus on optimizing nutrition, monitoring growth and quality of life, and limiting drug-related side effects [5].

IBD disease activity may be assessed using the activity indexes (AI) Pediatric Ulcerative Colitis Activity Index (PUCAI) and Pediatric Crohn's Disease Activity Index (PCDAI), which allow clinical symptoms, physical signs and biochemical markers to be converted into an activity score. These are reliable indicators of disease severity and remission status and are helpful in evaluating the response to treatment changes. An AI score of $<10$ accurately reflects clinical remission [6,7].

Thiopurines, such as azathioprine and mercaptopurine, are immunomodulatory agents recommended for the maintenance of steroid-free remission in children with IBD [4]. They are initiated soon after diagnosis and can take up to 14 weeks to be fully effective. The recommended starting doses are currently 2-2.5 mg/kg for azathioprine and 1-1.5 mg/kg for mercaptopurine $[4,8]$. 
Thiopurine use is associated with dose-dependent adverse drug reactions (ADRs), often leading to dose reduction or drug discontinuation [9]. Hematological toxicity in the form of myelosuppression, secondary to either thiopurine or elevated levels of its metabolite 6-thioguanine (6-TGN), is the most serious ADR. A review of 66 trials including 8302 patients reported a cumulative incidence of myelosuppression of $7 \%$, predominantly occurring within the initial few months of treatment $[10,14]$.

Although the use of thiopurine in the management of pediatric IBD is increasing and the measurement of the thiopurine metabolites 6-TGN and 6-methyl-mercaptopurine (6-MMP) can be helpful in determining compliance and optimizing therapy, these metabolites are not routinely monitored [11]. The cost and availability of the tests have been cited as reasons for this [10].

Thiopurine metabolite measurement is available at Evelina London Children's Hospital and is currently routinely used as a tool for monitoring children receiving thiopurine therapy. In this paper we describe a proactive approach, combining clinical data and thiopurine metabolite measurement, to guide azathioprine dosing in children with IBD.

\section{Patients and methods}

Pediatric IBD patients on azathioprine therapy were identified through a retrospective review of medical records between April 2015 and June 2017. Patients' demographics, disease phenotype, treatments and clinical data to establish disease AI were extracted from electronic medical records. Thiopurine methyltransferase (TPMT) status, 6-TGN and 6-MMP levels, blood count and inflammatory markers (C-reactive protein and erythrocyte sedimentation rate) were retrieved from the hospital's electronic records. The relevant disease-specific AIs were used (PCDAI and PUCAI).

Clinical remission was defined as a PCDAI or PUCAI $<10[12,13]$. Children with deficient or intermediate TPMT enzyme activity (normal range $26-50 \mathrm{pmol} / \mathrm{h} / \mathrm{mgHb}$ ) were excluded, as this affects azathioprine dosing. Children with very low $(<150) 6$-TGN levels due to lack of compliance with treatment were also excluded, although continued support was provided to develop strategies to improve adherence to medication.

The study was registered with and approved by the hospital review board; a full ethical review was not deemed to be necessary.

\section{Statistical analysis}

Statistical analysis was performed using IBM SPSS ${ }^{\circledR}$ Statistics Version 22. Data were expressed as mean \pm standard deviation (SD). Pearson's correlation coefficient was used to investigate the relationship between the variables. All statistical tests were 2 -tailed and a $\mathrm{P}$-value $<0.05$ was indicative of statistical significance.

\section{Results}

A total of 41 patients were identified, of whom 16 (40\%) were female and $25(60 \%)$ male. One patient was excluded as the initial TPMT value was $<26$. Of these identified patients, 12 had a diagnosis of ulcerative colitis (30\%), 26 with Crohn's Disease (65\%), and 2 with unclassified IBD (5\%). The mean age at diagnosis was $12.2 \pm 3.4$ years. Mean TPMT value was $41.8 \pm 11 \mathrm{pmol} / \mathrm{h} / \mathrm{mgHb}$. The azathioprine dose was $1.3 \pm 0.4 \mathrm{mg} / \mathrm{kg}$.

To achieve the initial induction of remission, prednisolone was used in 13 patients, exclusive enteral nutrition in 19 patients, and aminosalicylates in 4 . Four patients were treated in different institutions and their data were unavailable.

AI at time of starting therapy was $23 \pm 16$, reducing to 6 after 65 days (interquartile range [IQR] 29-85). Mean 6-TGN value was $280 \pm 151 \mathrm{pmol} / 8 \times 10^{8} \mathrm{red}$ blood cells $(\mathrm{RBC})$ and mean $6-\mathrm{MMP}$ value $1023 \pm 1008 \mathrm{pmol} / 8 \times 10^{8} \mathrm{RBC}$. The mean lymphocyte count was $1.7 \pm 0.8 \times 10^{9} / \mathrm{L}$. Twenty-eight children were in clinical remission with therapeutic 6-TGN, 12 had sub-therapeutic 6-TGN for which the azathioprine dose was increased. Twelve children were on 5 -aminosalicylate and 5 were on biologic therapy ( 4 on infliximab and 1 on adalimumab) (Table 1). Mean age was $12.4 \pm 3.4$ years, azathioprine dose $1.4 \pm 0.5 \mathrm{mg} / \mathrm{kg}$, and $\mathrm{AI}$ at the start of azathioprine $23 \pm 14$.

After 61 (IQR 27-74) days, levels of 6-TGN were $265 \pm 150 \mathrm{pmol} / 8 \times 10^{8} \mathrm{RBC}, 6-\mathrm{MMP} 1112.3 \pm 1073.4 \mathrm{pmol} / 8 \times$ $10^{8} \mathrm{RBC}$, white blood cells (WBC) $8.2 \pm 3.4 \times 10^{9} / \mathrm{L}$, lymphocytes $1.8 \pm 0.9 \times 10^{\%} / \mathrm{L}$, neutrophils $5.1 \pm 3.2 \times 10^{9} / \mathrm{L}$ and the $\mathrm{AI}$ at the time of measurement was $5 \pm 5$ (Table 2 and Fig. 1). At the time of metabolite measurement, all children were on maintenance therapy having completed standard induction.

Table 1 Background information

\begin{tabular}{|c|c|}
\hline Characteristic & Value \\
\hline Total patients & 40 \\
\hline Females (percent) & $16(40 \%)$ \\
\hline Age (years $\pm \mathrm{SD}$ ) & $12.2 \pm 3.4$ \\
\hline Ulcerative colitis & $12(30 \%)$ \\
\hline Crohn's disease & $26(65 \%)$ \\
\hline Unclassified inflammatory bowel disease; & $2(5 \%)$ \\
\hline Azathioprine dose $\mathrm{mg} / \mathrm{kg}($ mean $\pm \mathrm{SD})$ & $1.3 \pm 0.4$ \\
\hline Time metabolites measured (days \pm SD) & $65 \pm 45$ \\
\hline White blood cells $\times 10^{9}($ mean \pm SD $)$ & $8.1 \pm 3.1$ \\
\hline Lymphocytes $\times 10^{9}($ mean \pm SD $)$ & $1.7 \pm 0.8$ \\
\hline Neutrophils $\times 10^{9}($ mean $\pm \mathrm{SD})$ & $5.1 \pm 3.1$ \\
\hline Children on infliximab & 4 \\
\hline Children on adalimumab & 1 \\
\hline
\end{tabular}




\section{Discussion}

Thiopurines such as azathioprine and mercaptopurine are immunomodulatory agents recommended for the maintenance of steroid-free remission in children with IBD [4]. Once absorbed, azathioprine is converted into its active metabolites, 6-MMP and 6-TGN, via an enzymatic pathway involving TPMT $[9,8]$.

A therapeutic 6-TGN range is defined as $230-450 \mathrm{pmol} / 8 \times 10^{8} \mathrm{RBC}$. Interpretation of the range as low $(<230)$ or high $(>450)$ depends on the clinical features. In cases of active disease, a low or absent 6-TGN level may indicate nonadherence to medication or under-dosing, in which case patient education or a dose increase is indicated [14]. A low or normal 6-TGN level and a high 6-MMP level may indicate thiopurine intolerance, and the dose should be split: e.g. twice daily or allopurinol added as an adjunct to low-dose thiopurine [11]. A high 6-TGN level with active disease suggests thiopurine refractory disease, and alternative treatment should be sought $[10,4]$. This study evaluated a cohort of pediatric patients with IBD who achieved therapeutic thiopurine metabolites with lower than the target recommended azathioprine dose. Blood tests were performed according to current guidelines and azathioprine metabolites were measured 6 weeks after the initiation of therapy. The azathioprine dose was adjusted to achieve therapeutic metabolite levels.

To maintain therapeutic 6-TGN levels, the effective azathioprine dose for the whole group in this study was $1.3 \pm 0.4 \mathrm{mg} / \mathrm{kg}$, as there is emerging evidence to suggest a lower than standard azathioprine dose in patients receiving combination therapy with biologics [15]. Excluding the children on combination therapy, the effective azathioprine dose in children was $1.4 \pm 0.5 \mathrm{mg} / \mathrm{kg}$ (Table 2). Similarly to previous reports, we were able to achieve clinical remission, as measured by disease AI (PUCAI and PCDAI), in children with a mean 6 -TGN of $260 \pm 151 \mathrm{pmol} / 8 \times 10^{8} \mathrm{RBC}$ (in the whole cohort) under azathioprine monotherapy. None of the children developed azathioprine toxicity as defined by myelosuppression or abnormal liver function tests. The azathioprine dose was not changed in 28 children who were in clinical remission and had therapeutic 6-TGN levels, while in 12 children 6-TGN levels were sub-therapeutic and their azathioprine dose was increased. We did not observe a significant correlation between azathioprine (monotherapy) dose and 6-TGN levels ( $\mathrm{r}=0.3$, $\mathrm{P}=0.20$ ) or a significant correlation between $\mathrm{WBC}$ and TGN levels ( $\mathrm{r}=-0.18, \mathrm{P}=0.3$; Fig. 2,3 ). This may be due to the small sample size, as those correlations have been well documented in other studies.

Reduced enzyme activity results in an increased risk of myelosuppression. It is therefore recommended to check TPMT levels in every child prior to the initiation of therapy (normal range $25-50 \mathrm{pmol} / \mathrm{h} / \mathrm{mgHb}$ ). Cytopenia can occur even in the presence of normal TPMT activity; therefore, blood monitoring is required in all patients [4]. Dose reduction or alternative treatments can be considered where an abnormal TPMT status is identified [8].

Side effects and ADRs, including gastroenterological disturbance, skin rash and pancreatitis, are common

Table 2 Excluding children on biologics

\begin{tabular}{lc}
\hline Characteristic & Mean \pm SD \\
\hline Age (years) & $12.4 \pm 3.4$ \\
Azathioprine dose $(\mathrm{mg} / \mathrm{kg})$ & $1.4 \pm 0.5$ \\
Time metabolites measured (days) & $61 \pm 45$ \\
White blood cells $\times 10^{9}(/ \mathrm{L})$ & $8.1 \pm 3.4$ \\
Lymphocytes $\times 10^{9}(/ \mathrm{L})$ & $1.8 \pm 0.9$ \\
Neutrophils $\times 10^{9}(/ \mathrm{L})$ & $5.1 \pm 3.2$ \\
\hline SD, standard deviation & \\
\hline
\end{tabular}

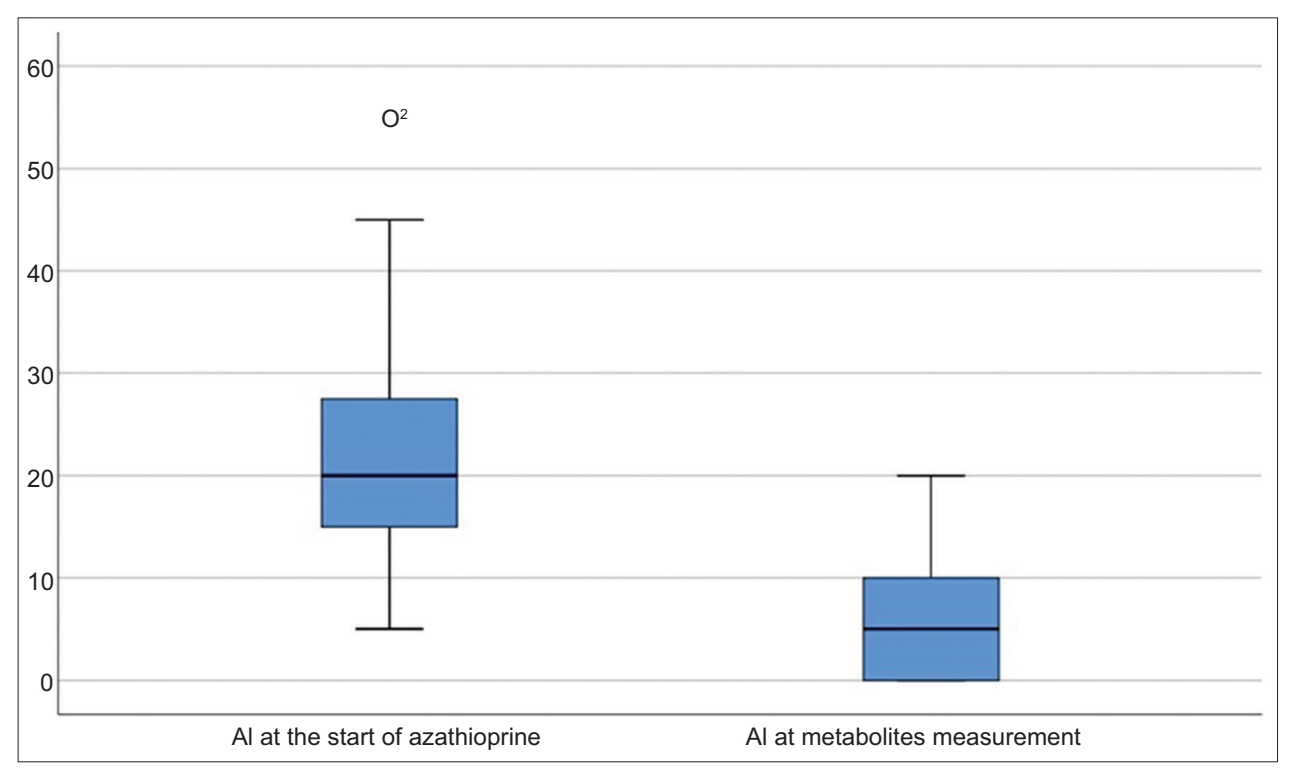

Figure 1 Activity index (AI) at the start of azathioprine treatment and at the time of metabolite measurement 


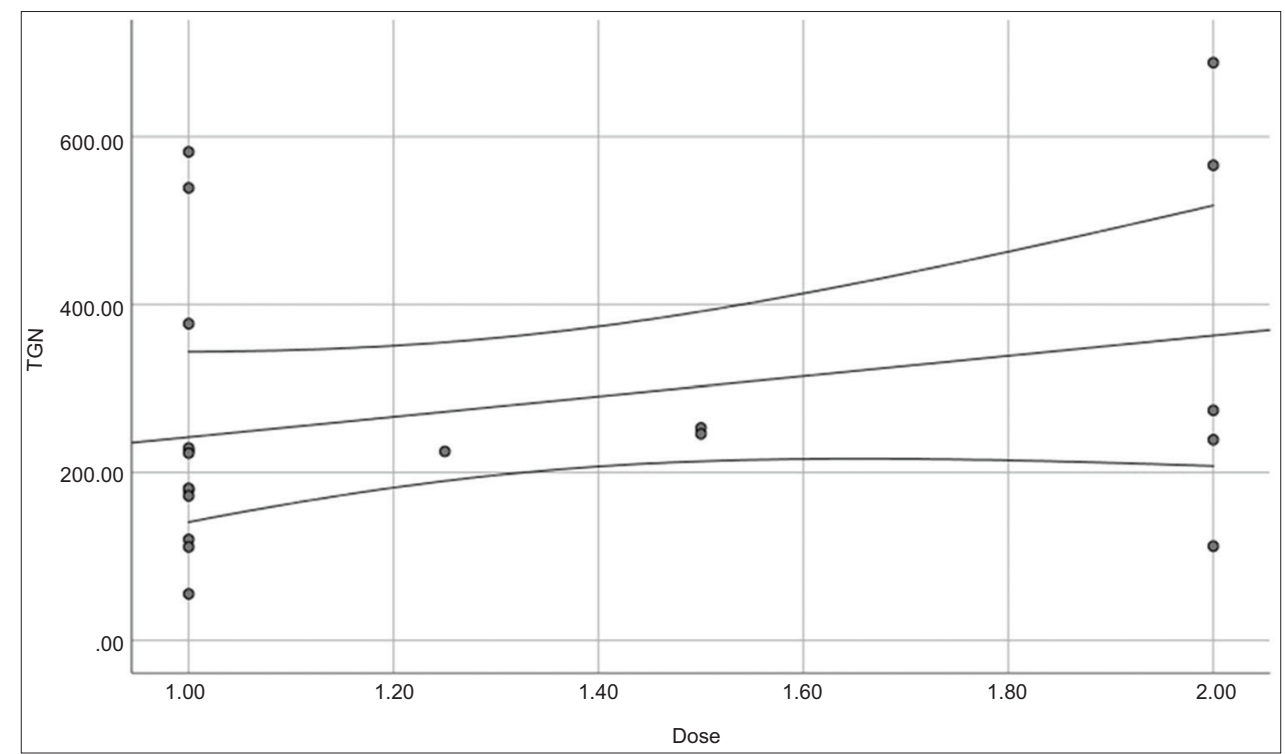

Figure 2 Scatterplot for azathioprine dose and thioguanine (TGN) level $(\mathrm{r}=0.3 \mathrm{P}=0.20)$

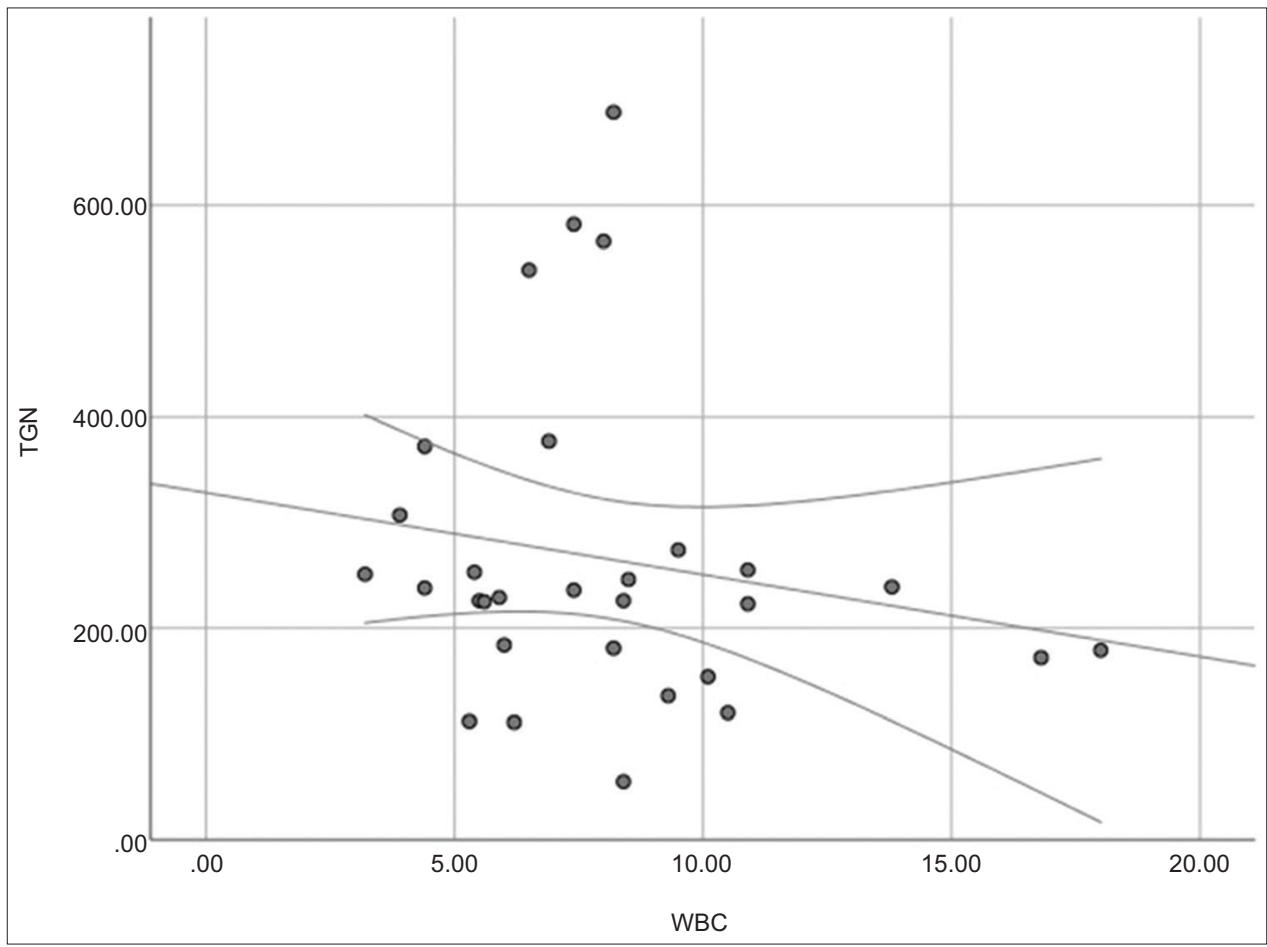

Figure 3 Scatterplot for white blood cell (WBC) counts and thioguanine (TGN) level ( $r=-0.18 \quad \mathrm{P}=0.3$ )

with thiopurine treatment, requiring dose adjustment or discontinuation in up to $25 \%$ of adult patients [16], with $56 \%$ of ADRs occurring within 1 month and $92 \%$ within 3 months of treatment. A multifaceted approach is required to maximize the response to and safety of these drugs. Pre-treatment TPMT status and regular monitoring of blood counts on treatment is considered the minimum standard for patients treated with thiopurine. Using 6-TGN levels above $235 \mathrm{pmol} / 8 \times 10^{8}$ $\mathrm{RBC}$ as a surrogate marker of efficacy is well documented and is associated with maintaining clinical remission [17]. It is also important to minimize the idiosyncratic side-effects by using an appropriate dosing regimen. Although the current dosing regimen for thiopurine therapy is body-weight based, there is increasing evidence to support the use of metabolite measurement to adjust thiopurine doses. Similarly to our findings, Hibi et al reported that low-dose thiopurine was effective and safe in maintaining remission in adults with ulcerative colitis [18]. They described a significantly lower 
incidence of myelosuppression in the low-dose cohort compared with the standard-dose group.

6-TGN levels have been shown to increase with thiopurine dose escalation and decrease with dose reduction, and a study by Nguyen et al explored this relationship, the results of which support the use of metabolite levels to reliably inform and guide thiopurine dosing [9]. 6-TGN levels are also useful in identifying cases of non-adherence to thiopurine therapy, and it has been suggested that routine observation of the metabolites can help in improving adherence rates [14]. A further study demonstrated that the majority of patients had a favorable outcome when their treatment was directed by metabolite measurement and that this can lead to better optimization of thiopurine treatment [19].

A subjectively reported barrier to routine metabolite measurement was the cost of the test and reimbursement issues; however, as demonstrated by Dubinsky et al, the overall cost may be reduced [13]. In children who find the side-effects of thiopurine therapy intolerable, the next treatment step, such as biologics, can be costly.

The data collected from this cohort show that, in a subset of children with IBD, early monitoring of thiopurine metabolites can aid clinical decisions regarding azathioprine dosing, and suggests that therapeutic thiopurine metabolites can be achieved with lower than the recommended target dose.

We acknowledge the limitations of this study, including the small sample size and retrospective design. We also acknowledge the short follow-up period, and that some children could still be experiencing the effect of induction therapy, making it difficult to comment on the effect of azathioprine alone and therefore to extrapolate any assumptions on safety. Therefore, we do not suggest replacing weight-based azathioprine dosing with metabolite measurement, but rather that this should be used as an adjunct to help optimize therapy, minimize unwanted adverse effects and assist in the timely guidance of clinical decision making.

\section{Summary Box}

\section{What is already known:}

- Thiopurines are standard treatment for children with inflammatory bowel disease (IBD)

- Weight-based dosing is the current recommended regime for thiopurine treatment in these patients

- Thiopurine metabolites are increasingly used to monitor treatment

\section{What the new findings are:}

- This study describes a proactive approach to dosing and monitoring children on thiopurine therapy

- This paper suggests standard weight-based dosing may not be applicable to children with IBD

\section{References}

1. Zhang YZ, Li YY. Inflammatory bowel disease: pathogenesis. World J Gastroenterol 2014;20:91-99.

2. Benchimol EI, Fortinsky KJ, Gozdyra P, Van den Heuvel M, Van Limbergen J, Griffiths AM. Epidemiology of pediatric inflammatory bowel disease: a systematic review of international trends. Inflamm Bowel Dis 2011;17:423-439.

3. Oliveira SB, Monteiro IM. Diagnosis and management of inflammatory bowel disease in children. BMJ 2017;357:j2083.

4. Ruemmele FM, Veres G, Kolho KL, et al. Consensus guidelines of ECCO/ESPGHAN on the medical management of pediatric Crohn's disease. J Crohns Colitis 2014;8:1179-1207.

5. Kammermeier J, Morris MA, Garrick V, Furman M, Rodrigues A, Russell RK; BSPGHAN IBD Working Group. Management of Crohn's disease. Arch Dis Child 2016;101:475-480.

6. Romano C, Syed S, Valenti S, Kugathasan S. Management of acute severe colitis in children with ulcerative colitis in the biologics era. Pediatrics 2016;137.

7. Hyams J, Markowitz J, Otley A, et al; Pediatric Inflammatory Bowel Disease Collaborative Research Group. Evaluation of the pediatric Crohn disease activity index: a prospective multicenter experience. J Pediatr Gastroenterol Nutr 2005;41:416-421.

8. Benmassaoud A, Xie X, AlYafi MM, et al. Su1416 Thiopurines in the management of Crohn's disease: safety and efficacy profile in patients with intermediate and normal thiopurine methyltransferase activity, a retrospective study. Gastroenterology 2014; 146:S-463-S-464.

9. Nguyen TV, Vu DH, Nguyen TM, Lachaux A, Boulieu R. Relationship between azathioprine dosage and thiopurine metabolites in pediatric IBD patients: identification of covariables using multilevel analysis. Ther Drug Monit 2013;35:251-257.

10. González-Lama Y, Gisbert JP. Monitoring thiopurine metabolites in inflammatory bowel disease. Frontline Gastroenterol 2016;7:301-307.

11. Chevaux JB, Peyrin-Biroulet L, Sparrow MP. Optimizing thiopurine therapy in inflammatory bowel disease. Inflamm Bowel Dis 2011;17:1428-1435.

12. Turner D, Griffiths AM, Walters TD, et al. Appraisal of the pediatric Crohn's disease activity index on four prospectively collected datasets: recommended cutoff values and clinimetric properties. Am J Gastroenterol 2010;105:2085-2092.

13. Turner D, Hyams J, Markowitz J, et al; Pediatric IBD Collaborative Research Group. Appraisal of the pediatric ulcerative colitis activity index (PUCAI). Inflamm Bowel Dis 2009;15:1218-1223.

14. Stocco G, Londero M, Campanozzi A, et al. Usefulness of the measurement of azathioprine metabolites in the assessment of non-adherence. J Crohns Colitis 2010;4:599-602.

15. Roblin X, Boschetti G, Williet N, et al. Azathioprine dose reduction in inflammatory bowel disease patients on combination therapy: an open-label, prospective and randomised clinical trial. Aliment Pharmacol Ther 2017;46:142-149.

16. Wong DR, Coenen MJ, Vermeulen SH, et al. Early assessment of thiopurine metabolites identifies patients at risk of thiopurineinduced leukopenia in inflammatory bowel disease. J Crohns Colitis 2017;11:175-184.

17. Goel RM, Blaker P, Mentzer A, Fong SC, Marinaki AM, Sanderson JD. Optimizing the use of thiopurines in inflammatory bowel disease. Ther Adv Chronic Dis 2015;6:138-146.

18. Hibi T, Naganuma M, Kitahora T, Kinjyo F, Shimoyama T. Lowdose azathioprine is effective and safe for maintenance of remission in patients with ulcerative colitis. J Gastroenterol 2003;38:740-746.

19. Haines ML, Ajlouni Y, Irving PM, et al. Clinical usefulness of therapeutic drug monitoring of thiopurines in patients with inadequately controlled inflammatory bowel disease. Inflamm Bowel Dis 2011;17:1301-1307. 